\title{
Elaboração e validação do protocolo de desprescrição do clonazepam em idosos
}

\section{Elaboration and validation of clonazepam deprescribing protocol for the elderly}

\section{Elaboración y validación del protocolo de desprescripción del clonazepam en ancianos}

\author{
André Oliveira Baldoni ${ }^{1} \oplus$, Priscilla Ferreira Zadra ${ }^{1}$, Luisa Gallo Vilar ${ }^{1} \odot$, Marcos Antonio Anacleto Junior ${ }^{1} \odot$, Ana Cristina de

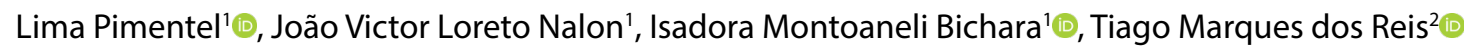 \\ ${ }^{1}$ Universidade Federal de São João Del Rei (UFSJ). Divinópolis, Minas Gerais, Brasil \\ ${ }^{2}$ Universidade Federal de Alfenas (UNIFAL-MG). Alfenas, Minas Gerais, Brasil
}

\section{Resumo}

Introdução: Algumas alterações fisiológicas que ocorrem no indivíduo idoso favorecem o acúmulo e a intoxicação por medicamentos. Dentre estes, podemos citar a classe dos benzodiazepínicos, medicamentos que, apesar de amplamente prescritos, principalmente para tratamento de distúrbios do sono e ansiedade, são considerados potencialmente inapropriados para o uso em idosos. Portanto, a elaboração de protocolos para desprescrição desses medicamentos é estratégia necessária na gestão do cuidado dos pacientes geriátricos. Objetivo: Elaborar e validar um protocolo de desprescrição do clonazepam para idosos que fazem uso deste medicamento para ansiedade ou insônia. Métodos: Estudo metodológico, desenvolvido em duas etapas, sendo elas a elaboração e a validação do protocolo de desprescrição do clonazepam para idosos que fazem uso desse medicamento para ansiedade ou insônia, excetuando-se aqueles que preenchem os critérios de exclusão. A elaboração do protocolo resultou em três produtos: um fluxograma de desprescrição, um folheto sobre higiene do sono e um folheto contendo os benefícios da desprescrição do clonazepam sob supervisão médica. A validação do protocolo foi realizada por médicos especialistas, por meio da Técnica de Delphi. Já na validação dos folhetos, participaram, além dos especialistas, indivíduos com 60 anos ou mais, de ambos os sexos, que não fizessem uso do clonazepam. A partir dos resultados obtidos, foi analisada a concordância da avaliação por meio do Coeficiente de Validade de Conteúdo (CVC), uma vez que essa ferramenta objetiva medir o grau de concordância dos juízes participantes do processo de validação. Resultados: O fluxograma foi considerado validado após a segunda rodada de avaliação, pois todos os itens avaliados obtiveram CVC igual ou superior a 0,8 nesta rodada. Os folhetos foram considerados validados já na primeira rodada de avaliação, pois todos os itens também obtiveram CVC superior a 0,8 durante esta rodada. Conclusão: Considerando os resultados obtidos, o protocolo se apresenta como uma ferramenta importante ao guiar a conduta médica no processo de desprescrição do clonazepam.

Palavras-chave: Protocolos. Desprescrições. Clonazepam. Idoso. Geriatria

Como citar: Baldoni AO, Zadra PF, Vilar LG, Anacleto Junior MA, Pimentel ACL, Nalon JVL, Bichara IM, Reis TM. Elaboração e validação do protocolo de desprescrição do clonazepam em idosos. Rev Bras Med Fam Comunidade. 2020;15(42):2105. https://doi.org/10.5712/rbmfc15(42)2105

\author{
Autor correspondente: \\ André De Oliveira Baldoni. \\ E-mail: andrebaldoni@ufsj.edu.br \\ Fonte de financiamento: \\ declaram não haver. \\ Parecer CEP: \\ Não se aplica. \\ Procedência e revisão por pares: \\ revisado por pares. \\ Recebido em: 12/06/2019. \\ Aprovado em: 17/10/2019.
}




\begin{abstract}
Introduction: Some physiological changes that occur in the elderly individual favor the accumulation and intoxication by drugs. Among these, we can mention the class of benzodiazepines, medicines which, although widely prescribed mainly for the treatment of sleep disorders and anxiety, are considered potentially inappropriate for use in the elderly. Therefore, the elaboration of protocols for the deprescribing of those drugs is a necessary strategy in the management of the care of geriatric patient. Objective: To elaborate and validate a protocol for the Deprescribing of clonazepam for the elderly who use this medication for anxiety or insomnia. Methods: Methodological study, developed in two stages - elaboration and validation of the protocol of deprescribing of clonazepam for elderly people who use this medication for anxiety or insomnia, except those who meet the exclusion criteria. The elaboration of the protocol resulted in three products: a flowchart of deprescribing, a leaflet of sleep hygiene, and a leaflet containing the benefits of the clonazepam Deprescribing under medical supervision. The validation of the protocol was performed by medical specialists, through the Delphi Technique. In addition to the specialists, individuals of both sexes, aged 60 years or more who did not use clonazepam, took part in the validation of the leaflets. Based on the results obtained, the agreement of the evaluation was analyzed using the Content Validity Coefficient (CVC), since this tool aims to measure the degree of agreement of the judges participating in the validation process. Results: The flowchart was considered validated after the second round of evaluation, since all items assessed had a CVC equal to or greater than 0.8 in this round. The leaflets were considered validated in the first evaluation round, since all items also obtained CVC greater than 0.8 during this round. Conclusion: Considering the results obtained, the protocol presents itself as an important tool in guiding medical conduct in the process of Deprescribing of clonazepam.
\end{abstract}

Keywords: Protocols. Deprescriptions. Clonazepam. Aged. Geriatrics

\title{
Resumen
}

Introducción: Algunas alteraciones fisiológicas que ocurren en el individuo mayor favorecen el acumulación y la intoxicación por medicamentos. Entre estas, podemos citar la clase de los benzodiazepínicos, medicamentos que, a pesar de ampliamente prescritos, principalmente para el tratamiento de disturbios del sueño y de la ansiedad, son considerados potencialmente inapropiados si usados por personas mayores. Por lo tanto, la elaboración de protocolos para desprescripción de esos medicamentos son estrategias necesarias en la gestión al cuidado de los pacientes geriátricos. Objetivo: Elaborar y validar un protocolo de desprescripción de clonazepam para mayores que hacen uso de esta medicina para ansiedad o insomnio. Métodos: Estudio metodológico, desarrollado en dos etapas, siendo ellas la elaboración y la validación del protocolo de desprescripción del clonazepam para mayores que hacen uso de ese medicamento para ansiedad o insomnio, exceptuando aquellos que cumplen los criterios de exclusión. La elaboración del protocolo resultó en tres productos: un flujograma de desprescripción, un folleto sobre la higiene del sueño y un folleto conteniendo los beneficios de la desprescripción del clonazepam bajo supervisión médica. La validación del protocolo fue realizada por médicos especialistas, por medio de la Técnica de Delphi. Ya en la validación de los folletos, participaron, además de los especialistas, individuos con 60 años o más, de ambos sexos, que no hiciesen uso de clonazepam. A partir de los resultados obtenidos, fue analizada la concordancia de la evaluación por medio del Coeficiente de Validez de Contenido (CVC), una vez que esa herramienta objetiva medir el grado de concordancia de los jueces partícipes del proceso de validación. Resultados: El flujograma fue considerado validado después de la segunda ronda de evaluación, pues todos los ítems evaluados obtuvieron CVC igual o superior a 0,8 en esta rodada. Los folletos fueron considerados validados ya en la primera rodada de evaluación, pues todos los ítems también obtuvieron CVC superior a 0,8 durante esta rodada. Conclusión: Considerando los resultados obtenidos, el protocolo se presenta como una herramienta importante al guiar la conducta médica en el proceso de desprescripción del clonazepam.

Palabras clave: Protocolos. Deprescripciones. Clonazepam. Anciano. Geriatría.

\section{Introdução}

Ao longo das últimas décadas, o Brasil passou a acompanhar uma transformação demográfica que culminou com o aumento da proporção de idosos na população. $\mathrm{O}$ avanço da medicina está na gênese desta transformação, reduzindo as taxas de mortalidade e de fecundidade e aumentando a expectativa de vida. ${ }^{1,2}$ Dentre as alterações fisiológicas que ocorrem no idoso, o declínio na função renal e hepática e o aumento da proporção de gordura corporal são as que mais possuem relação com a farmacologia clínica e com as alterações farmacocinéticas, sobretudo dos benzodiazepínicos. ${ }^{2,3}$ Essas alterações favorecem o acúmulo e a intoxicação por medicamentos. ${ }^{2}$ Os benzodiazepínicos são fármacos lipossolúveis que tendem a se acumular no tecido adiposo, prolongado sua meia vida de eliminação e acentuando seus efeitos sedativos. ${ }^{4}$

Diante desses fatos, existem "Medicamentos Potencialmente Inapropriados" (MPI) aos idosos, que são aqueles cujos benefícios são suprimidos pelos riscos de sua utilização, o que aumenta a probabilidade de ocorrência de efeitos adversos. ${ }^{5}$ Os MPI são definidos por critérios explícitos e identificados em instrumentos, como a lista de Beers. ${ }^{6}$ No Brasil, os estudos têm mostrado que os benzodiazepínicos estão entre os MPI mais utilizados pelos idosos, ${ }^{7}$ principalmente para o tratamento da insônia. ${ }^{8}$ 
No idoso, os efeitos adversos desses medicamentos tendem a ser mais pronunciados; dentre eles, destacam-se a sonolência, a diminuição da atenção, da coordenação motora, a amnésia anterógrada e a confusão mental. Estes efeitos podem aumentar o risco de queda e fratura óssea, e, assim, comprometer a segurança e qualidade de vida dos idosos. ${ }^{4}$ Diante disso, destaca-se a importância de medidas de intervenção para orientar a desprescrição desses medicamentos. Considera-se desprescrição como o processo de retirada de um medicamento inapropriado, em comum acordo com o paciente, supervisionado por um profissional de saúde para monitoramento dos efeitos benéficos ou prejudiciais da retirada deste medicamento. ${ }^{9}$

Na prática clínica, os benzodiazepínicos são amplamente utilizados por longo período de tempo. No entanto, estes medicamentos causam dependência física e psíquica que dificultam a interrupção do tratamento, mesmo quando há indicação clínica. ${ }^{7}$ Ademais, a literatura é escassa em relação às evidências disponíveis sobre o processo de desprescrição dessa classe de medicamentos.

Dentre os benzodiazepínicos mais comumente utilizados, destaca-se o clonazepam, considerado um benzodiazepínico de alta potência e meia vida longa, entre 18 e 50 horas, levando à potencialização dos seus efeitos a longo prazo, particularmente na população idosa. ${ }^{10}$ Entre o período de 2007 a 2010, o clonazepam foi o medicamento prescrito mais consumido no Brasil, considerando-se todos os medicamentos sujeitos a receituário especial. ${ }^{11}$

As medidas de intervenção para desprescrição de medicamentos podem ser guiadas através de protocolos, que são recomendações elaboradas sistematicamente, para facilitar o manejo de um problema de saúde, dentro de um contexto clínico específico, de preferência embasado em evidências científicas e na experiência clínica. Esses protocolos são ferramentas essenciais para padronização de condutas no exercício da prática médica. ${ }^{12}$ Diante disso, a elaboração de protocolos para desprescrição baseada em evidências e a utilização de medidas não farmacológicas, como orientações acerca da higiene do sono, são estratégias necessárias na gestão do cuidado dos pacientes geriátricos. ${ }^{13}$

Neste contexto, o presente estudo teve como objetivo elaborar e validar um protocolo de desprescrição do clonazepam para idosos que fazem uso deste medicamento para ansiedade ou insônia, considerando que este é o benzodiazepínico mais prescrito e utilizado pela população idosa de uma região do Centro-Oeste Mineiro. ${ }^{14}$

\section{Métodos}

Trata-se de um estudo metodológico desenvolvido em duas etapas: (I) elaboração do protocolo de desprescrição do clonazepam para idosos que fazem uso deste medicamento para ansiedade ou insônia; 8 e (II) validação do conteúdo do mesmo.

O protocolo é composto por um fluxo de estratégias para a desprescrição do clonazepam e dois folhetos educativos, um sobre a higiene do sono e outro sobre os benefícios da desprescrição do clonazepam sob supervisão médica, ambos a serem utilizados pelo médico no processo de desprescrição do clonazepam em idosos.

O protocolo contempla as ações necessárias para o processo de desprescrição. Ele foi desenvolvido baseando-se em uma revisão narrativa da literatura fundamentada em trabalhos que abordam listas de MPI aos idosos, ${ }^{5,15}$ utilização de terapias farmacológicas e não farmacológicas, como terapias cognitivocomportamentais, para facilitar a retirada de medicamentos, ${ }^{8,15-17}$ acompanhamento longitudinal e associação entre os diversos profissionais de saúde da atenção básica para auxílio durante o processo de desprescrição. ${ }^{18}$ 
A população que validou o protocolo foi constituída por médicos nas áreas da clínica médica, da geriatria, da Medicina de família e comunidade e da psiquiatria, de diversos municípios do Brasil, considerando a proximidade destas áreas de atuação com a população idosa. Os médicos foram contatados via e-mail e selecionados por meio de busca ativa dos seus endereços eletrônicos pela internet, telefone e participação em eventos científicos.

Em relação à validação dos folhetos, além dos médicos, participaram da avaliação da compreensão do conteúdo indivíduos com 60 anos ou mais, de ambos os sexos, usuários do Sistema Único de Saúde (SUS), que não estavam utilizando o medicamento clonazepam. A avaliação do conteúdo dos folhetos foi realizada por idosos que não utilizam o clonazepam com o intuito de assegurar que a desprescrição não ocorresse apenas com a leitura do material, sem acompanhamento médico, certificando os aspectos legais e éticos do estudo.

Coluci et al. ${ }^{19}$ recomendam que a avaliação de instrumentos na área da saúde seja feita por 30 a 40 indivíduos da população-alvo. Neste estudo, a população-alvo foi composta pelos médicos e pelos idosos. Para guiar essa avaliação, foi elaborado um instrumento contendo oito questões referentes ao fluxograma e oito questões referentes aos folhetos. As opções de resposta foram organizadas em uma escala de frequência tipo Likert, pontuada de zero a 10, sendo zero “discordo plenamente" e 10 "concordo plenamente". Após cada questão, foi inserido um campo opcional para comentários, críticas e sugestões dos especialistas. Aavaliação teve como finalidade julgar as questões técnicas do protocolo, a compreensão dos itens e a inteligibilidade das respostas propostas, averiguando-se o grau de entendimento e a necessidade de modificações. ${ }^{19}$

Os questionários de avaliação foram enviados por e-mail, por meio de formulário eletrônico disponível na plataforma Google Docs (https://www.google.com/forms/about/), em conjunto com o protocolo e o Termo de Consentimento Livre e Esclarecido (TCLE), inicialmente para 170 médicos, visto que, em média, somente $25 \%$ dos entrevistados respondem os questionários enviados à distância. ${ }^{20}$ Os especialistas tiveram um prazo de 14 dias para responder ao questionário.

Todos os dados coletados por meio de formulário eletrônico foram analisados e foi verificada a concordância entre as respostas dos juízes avaliadores por meio do Coeficiente de Validade de Conteúdo (CVC), proposto por Hernández-Nieto. ${ }^{21} \mathrm{O}$ objetivo deste coeficiente é medir esse grau de concordância em cada questão presente no questionário. ${ }^{21}$

Para cada item, foi calculado o CVC seguindo as seguintes etapas: (a) cálculo da média das notas (Mx); (b) cálculo do CVC inicial (CVCi), por meio da divisão da média pelo valor máximo que o item poderia alcançar; (c) cálculo do erro (Pei), a partir da divisão do número um (1) pelo total de juízes avaliadores, elevado pelo mesmo número de avaliadores - o erro tem como objetivo minimizar possíveis vieses destes juízes -; e (d) cálculo do CVC final (CVCc), a partir da subtração do CVCi pelo Pei. Foram considerados válidos os itens com CVCc maiores que 0,8, conforme proposto por Hernández-Nieto. ${ }^{21}$

Os tópicos que não atingiram a concordância estipulada foram reformulados com base nas opiniões e sugestões dadas pelos avaliadores no espaço disponibilizado para feedback contido no questionário, e, posteriormente, o material foi reenviado aos especialistas, iniciando uma nova etapa de avaliação. $O$ reenvio do questionário com as devidas reformulações pode ser feito quantas vezes for necessário, até que se obtenha o CVC desejado. Esta metodologia de aplicação de questionários por sucessivas etapas, em busca de um consenso de opiniões entre um grupo de especialistas consiste na Técnica de Delphi. 
Esse consenso que se busca, por meio da convergência de opiniões, resulta em um julgamento coletivo, que pressupõe-se ser melhor do que a opinião de um só indivíduo. ${ }^{22}$

Após a validação do conteúdo do protocolo pelos especialistas, os folhetos foram avaliados pelos idosos em relação à linguagem, compreensão do conteúdo, clareza e estrutura das informações por meio de outro instrumento contendo oito questões. As opções de resposta foram organizadas em uma escala de frequência tipo Likert, seguindo o mesmo padrão de avaliação realizado pelos especialistas. A concordância em relação à avaliação dos folhetos feita pelos idosos também foi analisada pelo cálculo do CVCc.

Os idosos foram abordados e convidados a participar da validação dos folhetos nas Unidades Básicas de Saúde, Estratégias de Saúde da Família e Centro de Especialidades Médicas do SUS em municípios de Minas Gerais, onde foram colhidas as assinaturas dos TCLE. Todo o processo contou com a presença dos pesquisadores para esclarecimento dos objetivos da pesquisa e de eventuais dúvidas que surgiram.

O trabalho foi aprovado pelo Comitê de Ética em Pesquisa Envolvendo Seres Humanos (CEPES) da Universidade Federal de São João del-Rei, sob o protocolo CAAE - 62370616.5.0000.5545.

\section{Resultados}

A elaboração do protocolo foi feita a partir de uma revisão narrativa da literatura que orienta a desprescrição de benzodiazepínicos em outros países e que serviu como base para a sistematização de um protocolo que pudesse ser utilizado no contexto brasileiro. Em sua primeira versão, o fluxograma foi elaborado de acordo com a sequência: estabelecimento de critérios de inclusão e exclusão de pacientes para participação do processo de desprescrição do clonazepam; convite realizado pelos médicos responsáveis aos pacientes que cumprissem com os critérios exigidos; consentimento do paciente em relação à sua participação; distribuição dos folhetos educativos e exposição verbal a respeito dos benefícios e riscos decorrentes da retirada desse medicamento; e instrução em relação à dose a ser reduzida, intervalo entre reduções e condutas a serem adotadas em caso de respostas negativas à redução proposta.

A construção dos folhetos de higiene do sono e da retirada gradual do clonazepam seguiu recomendações do Consenso Brasileiro de Medicamentos Potencialmente Inapropriados para Idosos, que aborda, com base nos critérios de Beers e STOPP, a utilização de medicamentos e seus diversos efeitos colaterais apresentados nessa população. ${ }^{5}$

Com foco no clonazepam, medicamento amplamente utilizado em território nacional, são relatados os principais efeitos colaterais esperados quando iniciada a retirada gradual deste medicamento. Esses dados, explicitados através de linguagem simples e imagens ilustrativas, têm o intuito de alertar o paciente sobre os sintomas que podem surgir, tranquilizar quanto à sua origem e orientar a busca de apoio à equipe de saúde que realiza a desprescrição quando necessário.

O folheto de higiene do sono foi construido de acordo com recomendações da Sociedade Brasileira do Sono, e contempla orientações sobre hábitos de vida modificáveis, que contribuem para uma melhor qualidade do sono. Enfatiza a prática de atividade física, atividades relaxantes e a regularidade nos hábitos de sono diários. O folheto foi elaborado com vocabulário simplificado, apresentando imagens relacionadas às informações, facilitando o entendimento das orientações do mesmo para usuários com escolaridade distinta. 
Estes produtos, então, foram enviados aos juízes avaliadores, dando início à etapa de validação do fluxograma e dos folhetos, de acordo com a metodologia apresentada anteriormente. Após o envio dos 170 convites para participação do estudo, foram obtidas 43 respostas durante a primeira etapa de avaliação do protocolo. O perfil dos médicos participantes nesta etapa foi predominantemente de especialistas em Medicina de família e comunidade (53\%).

Os avaliadores concluíram o curso de Medicina entre os anos de 1986 e 2017, sendo que 53\% se graduaram entre 1 e 5 anos atrás. Na segunda etapa de avaliação, o protocolo foi reenviado para os 43 participantes da primeira etapa, sendo obtidas 13 repostas e a Medicina de família e comunidade continuou como a especialidade predominante entre os avaliadores (39\%). Os profissionais participantes da segunda etapa concluíram o curso de Medicina entre os anos de 2000 e 2016 (Tabela 1).

Em relação à avaliação do fluxograma, quatro dos oito itens (50\%) não obtiveram um CVCc igual ou superior a 0,8 durante a primeira etapa (Tabela 2). Portanto, o fluxograma foi reformulado de acordo com as sugestões dos especialistas para realização da segunda etapa do processo (Quadro 1).

Tabela 1. Perfil dos especialistas participantes da validação do protocolo de desprescrição do clonazepam em idosos.

\begin{tabular}{|c|c|c|}
\hline & Primeira Etapa $(n=43)$ & Segunda Etapa $(n=13)$ \\
\hline \multicolumn{3}{|l|}{ Especialidade Médica } \\
\hline Medicina de Família e Comunidade & 23 & 5 \\
\hline Geriatria & 6 & 1 \\
\hline Psiquiatria & 6 & 3 \\
\hline Clínica Médica & 5 & 2 \\
\hline Generalista & 3 & 2 \\
\hline \multicolumn{3}{|l|}{ Tempo de formação } \\
\hline $1-5$ anos & 23 & 7 \\
\hline 6-10 anos & 8 & 3 \\
\hline 11-15 anos & 5 & 2 \\
\hline 16 anos ou mais & 7 & 1 \\
\hline
\end{tabular}

Tabela 2. Coeficiente de validade de conteúdo final (CVCc) das avaliações dos especialistas sobre o conteúdo do fluxograma e dos folhetos.

\begin{tabular}{lcc}
\hline \multicolumn{1}{c}{ Itens Referentes ao Fluxograma } & \multicolumn{2}{c}{ CVCc } \\
\cline { 2 - 3 } & Primeira Etapa (N=43) & Segunda Etapa (N=13) \\
\hline Clareza, simplicidade e inequivocidade do fluxo & 0,834 & 0,923 \\
Coerência na sequência das ações & 0,869 & 0,876 \\
Itens expressando uma única ideia & 0,86 & 0,915 \\
Práticas baseadas em evidências mais atuais & 0,872 & 0,869 \\
Aplicabilidade à realidade dos idosos & 0,769 \\
Possibilidade de fazer a desprescrição sem a inserção de novos medicamentos & 0,632 \\
Aplicabilidade da redução da dose do clonazepam nos valores propostos & 0,713 \\
Viabilidade da aplicação do protocolo na prática clínica & 0,797 \\
\hline \multicolumn{1}{c}{ Itens Referentes aos Folhetos } & \\
\hline Adequação da linguagem & 0,886 \\
Relevância das informações & 0,916 \\
Compreensão do tema através do texto e ilustrações & 0,911 \\
Clareza e objetividade das mensagens & 0,895 \\
Sequência lógica do conteúdo & 0,895 \\
Adequabilidade do material ao nível sociocultural do público-alvo & 0,825 \\
Adequabilidade do tamanho do título e tópicos & 0,838 \\
Contribuição dos folhetos para o processo de desprescrição & 0,848 \\
\hline
\end{tabular}


Em relação aos folhetos, todos os itens obtiveram CVCc superior a 0,8 durante a primeira etapa de avaliação pelos especialistas. No entanto, alguns tópicos foram reformulados de acordo com os comentários enviados com o objetivo de melhorar a compreensão do conteúdo, como o aumento do número de figuras autoexplicativas e a troca do título do folheto de "Desprescrição do Clonazepam" para "Retirada Gradual do Clonazepam".

Durante a segunda etapa de avaliação do fluxograma, todos os itens obtiveram CVCc igual ou superior a 0,8 . No entanto, uma resposta considerada outlier foi excluída, pois atribuiu nota 0 a um dos itens sem nenhuma justificativa ou sugestão de mudança. Além disso, a pergunta acerca da viabilidade da realização da desprescrição sem a inserção de novos medicamentos ao plano terapêutico foi retirada da segunda etapa da avaliação, considerando que o fluxograma foi modificado inserindo-se a classe dos inibidores seletivos da recaptação da serotonina (ISRS), segundo as sugestões dos especialistas, durante a primeira rodada de avaliação.

Em relação aos folhetos, participaram da validação 35 idosos, conforme recomenda Coluci et al. ${ }^{19}$ Todos os itens mantiveram o CVCc superior a 0,8 durante a segunda etapa de avaliação pelos especialistas (Tabela 2).

$\mathrm{Na}$ avaliação dos folhetos pelos idosos, todos os itens obtiveram CVCc superior a 0,8 durante a primeira etapa (Tabela 3). No entanto, optou-se por adequar os folhetos de acordo com sugestões feitas pelos idosos no sentido de melhorar o layout dos mesmos.

Os produtos do protocolo - fluxograma e folhetos - estão disponíveis na Internet e podem ser visualizados na plataforma Open Science Framework (OSF) ${ }^{23}$

Quadro 1. Sugestões realizadas pelos especialistas durante a primeira rodada da validação do protocolo.

\begin{tabular}{lc}
\multicolumn{1}{c}{ Sugestão } & Sugestão acatada pelos pesquisadores (Sim/Não) \\
\hline Padronização seguindo a normatização internacional de fluxogramas & Sim \\
Melhor caracterização dos critérios de inclusão dos pacientes aptos à desprescrição & Sim \\
Explicitação da equivalência entre as diferentes formas farmacêuticas do clonazepam & Sim \\
Adição de itens relativos ao envolvimento da equipe de saúde no processo de desprescrição & Sim \\
Indicação de inclusão dos pacientes em grupos de apoio & Sim \\
Inclusão de medicamentos como terapia de apoio à redução dos sintomas de abstinência & Sim \\
Aumento do intervalo de tempo entre as reduções das doses & Sim \\
Redução da dose de maneira mais gradativa & Sim \\
\hline
\end{tabular}

Tabela 3. Coeficiente de validade de conteúdo final (CVCc) dos dois folhetos na etapa de avaliação pelos idosos.

\begin{tabular}{|c|c|c|}
\hline \multirow{2}{*}{ Itens referentes aos folhetos } & \multicolumn{2}{|l|}{ CVCc } \\
\hline & Folmeto Benefícios da Retirada do Clonazepam (N=35) & FolHeto Higiene do Sono ( $\mathrm{N}=35$ ) \\
\hline Capa do folheto chama atenção do leitor & 0,931 & 0,968 \\
\hline Fonte com tamanho adequado & 0,945 & 0,96 \\
\hline Sequência adequada & 0,98 & 0,971 \\
\hline Compreensão do que foi apresentado & 0,934 & 0,962 \\
\hline Figuras ajudaram no entendimento do conteúdo & 0,974 & 0,965 \\
\hline Motivação para ler o folheto até o final & 0,96 & 0,974 \\
\hline Entendimento do folheto por qualquer adulto & 0,90 & 0,931 \\
\hline $\begin{array}{l}\text { Ajuda do folheto no processo de interrupção do uso } \\
\text { do clonazepam, supervisionado por um médico* }\end{array}$ & 0,948 & - \\
\hline $\begin{array}{l}\text { Possibilidade de adoção de hábitos mais saudáveis } \\
\text { com o uso do folheto** }\end{array}$ & - & 0,971 \\
\hline
\end{tabular}




\section{Discussão}

Os benzodiazepinicos são a classe de psicotrópicos mais prescritos na prática clínica, principalmente para o tratamento de insônia, transtornos de ansiedade e epilepsia, devido à sua ação ansiolítica, hipnótica, anticonvulsivante e relaxante muscular. O seu uso não é indicado por um período superior a 3 meses, especialmente em idosos, pois quando feito de maneira prolongada aumenta o risco de desenvolver dependência e outros efeitos adversos como sonolência, aumento do risco de quedas, diminuição da atenção e da coordenação motora. Apesar das recomendações da literatura, os benzodiazepínicos são utilizados frequentemente de forma inadequada, sem que haja supervisão médica para retirada gradual e suspensão da medicamento. ${ }^{24}$

Muitos estudos que tratam da redução gradual da dose de benzodiazepínicos sugerem realizar a desprescrição através de porcentagens dos medicamentos. ${ }^{8,16,18}$ No entanto, quando se trata de medicamentos cuja apresentação se dá na forma de comprimidos, a redução em porcentagens não é viabilizada na totalidade dos casos, devido à impossibilidade de repartição do medicamento em muitos fragmentos. Assim, é necessário escolher uma dose pré-determinada para redução do medicamento que possibilite atender a todas as formas de apresentação disponíveis, principalmente quando se trata da forma em comprimidos. No caso do município estudado, o clonazepam é o benzodiazepínico mais dispensado e, por isso, decidiu-se por padronizar o fluxograma utilizando este medicamento, visto que não seria possível orientar a desprescrição de todos os benzodiazepínicos em uma dose pré-determinada utilizando um único fluxograma.

Sendo assim, o presente estudo produziu um instrumento que sistematiza a retirada gradual do clonazepam e considera possíveis limitações durante o processo, bem como alternativas para enfrentamento destas. A literatura nacional apresenta algumas recomendações para a retirada gradual do clonazepam em idosos, porém não há um fluxograma para a desprescrição do medicamento de forma sistematizada e aplicável na prática clínica. ${ }^{8}$ Além disso, foram consideradas as particularidades do sistema de saúde brasileiro em realizar atendimentos de forma longitudinal e gratuita, assim como as diferentes formas de apresentação do clonazepam disponíveis no SUS. Portanto, o protocolo elaborado incluiu as adaptações necessárias para facilitar sua ampla utilização pelos profissionais de saúde tanto no contexto público quanto no privado.

Aatenção básica possui como fundamentos e diretrizes a assistência multiprofissional, interdisciplinar e em equipe. Isso possibilita a atenção integral ao usuário e a coordenação de seu cuidado na rede de atenção à saúde ${ }^{25}$ Assim, foi inserida no início do fluxograma a orientação de realizar palestras visando a sensibilização da equipe de saúde, através da reflexão sobre a importância da atuação de cada membro na integração do cuidado para obter êxito no processo de desprescrição do clonazepam. ${ }^{26}$

A literatura comprova que as diversas técnicas para retirada dos benzodiazepínicos, incluindo o clonazepam, são mais efetivas quando o paciente participa ativamente do processo. O cuidado centrado no paciente e a tomada de decisões conjuntas apresentam melhorias na satisfação, adesão, qualidade de vida e redução do tempo de tratamento quando comparados às decisões realizadas unilateralmente..$^{15}$ Neste protocolo optou-se por realizar um encontro inicial com o paciente no qual é descrito como será o processo de desprescrição e são expostos todos os possíveis efeitos da retirada do medicamento e a conduta a ser tomada, frente a esses sintomas. 
Outra forma de tornar mais efetiva a participação do paciente no processo é a elaboração e utilização de folhetos educativos. Seu emprego na área da saúde mostra-se como um amplificador dos objetivos e orientações a serem seguidas, levando, através de linguagem clara e objetiva, imagens e recursos didáticos, informações úteis àquela população alvo. A literatura descreve que as orientações em recursos educativos devem ser lidas, entendidas e lembradas com facilidade, para que sua efetividade seja alcançada. ${ }^{26}$

Com relação às sugestões feitas pelos especialistas referentes ao fluxograma na primeira etapa de avaliação, grande parte delas foram acatadas, uma vez que se mostraram pertinentes para o aprimoramento do constructo. A escolha pela padronização do fluxograma segundo a American National Standards Institute justifica-se por ser um sistema mundialmente reconhecido e utilizado. De acordo com Harrington, ${ }^{27}$ este tipo de fluxograma objetiva trazer uma compreensão mais detalhada de um processo por meio de símbolos padronizados.

Outra sugestão feita pelos especialistas consistiu na inclusão do clonazepam em gotas no protocolo, pois antes abordava somente a forma em comprimidos. Essa modificação foi fundamental para facilitar a desprescrição em todos os usuários que se enquadrassem dentro dos critérios de inclusão, independentemente da forma de apresentação do medicamento utilizado. Ressalta-se que a escolha em relação ao uso da forma em gotas ou em comprimidos envolve a decisão médica e a disponibilidade desses medicamentos no serviço de saúde.

Em relação à definição da dose para a redução do medicamento e intervalo de tempo entre reduções, utilizou-se como base os estudos de Pottie et al., ${ }^{8}$ Voshaar et al. ${ }^{17} \mathrm{e} \mathrm{Lader} \mathrm{et} \mathrm{al.}{ }^{18}$ De acordo com esses autores, a periodicidade de 14 dias entre as reduções das doses acarretou menor tempo de duração do processo de desprescrição; maiores índices de sucesso; e menores índices de descontinuação do processo. ${ }^{17}$

Apesar da dose proposta pelos mesmos autores para a redução inicial do benzodiazepínico ser de $25 \%$ em relação à dose anterior, optou-se por padronizar a retirada de $0,5 \mathrm{mg}$ a cada intervalo de redução, levando-se em consideração a disponibilidade do clonazepam na Relação Municipal de Medicamentos (REMUME) do local de estudo, que se encontra na forma de comprimido de $2 \mathrm{mg}$. No Brasil, esse medicamento possui duas subdivisões, o que permite o fracionamento em até quatro partes, com o uso de um cortador de comprimidos, sem resultar em importantes variações da dose de cada fração ingerida.

A literatura também traz a possibilidade de modificar a porcentagem de redução do medicamento para $12,5 \%$ ao final do processo, a fim de diminuir os sintomas de abstinência. ${ }^{8}$ No entanto, pela impossibilidade de partição do medicamento, optou-se por não incorporar essa orientação ao protocolo. Dessa forma, uma redução de $0,5 \mathrm{mg}$ do clonazepam a cada 14 dias está em conformidade com a literatura, que apresenta resultados satisfatórios no processo de desprescrição, e adequada à disponibilidade do medicamento pelo SUS.

Diante disso, não foram acatadas duas sugestões feitas pelos especialistas, pois, em ambas, a literatura respalda as informações contidas no fluxograma. A primeira delas diz respeito ao aumento do intervalo de tempo entre as reduções das doses, visto que sugeriu-se um tempo maior que 14 dias a cada redução; e a segunda se refere à diminuição da dose a cada redução inferior a $0,5 \mathrm{mg}$ ou 5 gotas do medicamento.

A redução em $25 \%$ da dose inicial do medicamento foi testada em alguns estudos, 8,18 indicando menor tempo de duração do processo de retirada, mas com maiores índices de sintomas de abstinência durante o processo. Para contrapor esta dificuldade, é indicado o uso de medicações adjuvantes. ${ }^{16}$ 
Além disso, outra dificuldade que impede a redução inferior a 0,5 mg do medicamento é o fracionamento do comprimido pelos usuários. Essa dificuldade poderia ser solucionada se todos os usuários que utilizassem a forma em comprimidos substituissem pela forma em gotas. No entanto, sabe-se que uma parcela da população brasileira não teria condições financeiras de adquirir o medicamento, visto que a solução em gotas não é disponibilizada em todo o Brasil. Sendo assim, além do respaldo da literatura, ${ }^{8,17,18}$ optou-se por manter a dose de $0,5 \mathrm{mg}$ ou 5 gotas do medicamento a cada redução, para viabilizar a desprescrição do clonazepam em todo território nacional.

No que se refere às possibilidades para superar possíveis problemas que poderiam prejudicar a continuidade da desprescrição, como a presença de sintomas de abstinência da medicação ou o retorno da insônia, na primeira versão do fluxograma orientou-se somente retornar à dose do medicamento utilizada anteriormente ao início dos sintomas. Contudo, estudos demonstram que sintomas de abstinência intensos compõem grande parcela dos casos de falha na retirada gradual dos benzodiazepínicos. ${ }^{16}$ Dentre estes sintomas, os mais frequentes são insônia moderada a grave, depressão, convulsões, alucinações e prejuízos motores. ${ }^{28}$

Uma das sugestões feitas pelos especialistas durante a validação do protocolo foi a inclusão de medicamentos como terapia de apoio à redução dos sintomas de abstinência. A literatura mostra que a utilização de medicamentos adjuvantes, com baixa incidência de dependência, como os inibidores seletivos da recaptação da serotonina (ISRS), pode levar a benefícios no manejo dos sintomas de abstinência dos benzodiazepínicos e a uma maior taxa de sucesso da desprescrição, em comparação à descontinuação sem o uso de medicamentos adjuvantes. ${ }^{29}$

Além disso, essa classe de medicamentos é considerada como a primeira linha para tratamento dos distúrbios do sono associados a transtornos ansiosos ${ }^{8}$. Portanto, a utilização de outros medicamentos durante o processo de desprescrição pode promover melhorias na qualidade do sono e controle da ansiedade nesses pacientes. ${ }^{30}$

Além disso, manteve-se a possibilidade de retornar à dose anterior utilizada, com o objetivo de evitar sintomas graves, e então alternar os dias de uso do medicamento, levando a uma maior tolerabilidade. A retirada nos estágios iniciais do processo geralmente é mais tolerável, enquanto os estágios finais tendem a ser mais sintomáticos. É de suma importância haver flexibilização e ajustes dinâmicos durante todo o tratamento do paciente, sem que haja um prolongamento excessivo da retirada (mais de 24 semanas). ${ }^{18}$

A incorporação das sugestões dos especialistas e dos idosos, por meio da Técnica de Delphi, possibilitou alcançar um consenso em relação à forma de desprescrever o clonazepam em idosos. Outros estudos também empregaram como parte dessa técnica o CVC para validar o instrumento e seus itens, uma vez que ele consegue medir a proporção de juízes que estão em concordância sobre tais itens, e, com isso, verificar a capacidade em medir com precisão o fenômeno a ser estudado. ${ }^{31,32} \mathrm{O}$ instrumento passou por modificações até que fosse possível obter a versão final validada, o que reforça a necessidade desta técnica para a elaboração de um protocolo de qualidade. ${ }^{28}$

A literatura descreve o nível de consenso necessário para validação de instrumentos da área de saúde pela técnica de Delphi entre $50 \%$ a $80 \%$, com média de duas a três etapas para a conclusão do processo de validação. O número de especialistas que perdem o seguimento varia entre $30 \%$ e $50 \%$ no primeiro ciclo, e $20 \%$ a $30 \%$ no segundo. ${ }^{33}$ Neste estudo optou-se por alcançar um consenso igual ou superior a $80 \%$, de acordo com o CVC. 
A validação do protocolo contou com a participação de profissionais de diversas especialidades médicas e, com isso, pôde considerar as diferentes perspectivas desses profissionais no cuidado do paciente idoso, de forma a valorizar as opiniões e experiências de cada um. De acordo com a literatura, é de extrema importância a multidisciplinaridade dos especialistas que realizam a avaliação na técnica de Delphi, pois permite o trabalho em equipe, a valorização das opiniões e perspectivas diversas sobre um tema. ${ }^{34}$

A atuação dos idosos no processo de validação dos folhetos foi oportuna para a identificação de pontos de difícil compreensão e algumas questões estéticas a serem melhoradas. Existe consenso na literatura de que o material de educação em saúde deve ser elaborado de forma simples e que permita transmitir informações exatas. As ilustrações devem ser atrativas e remeter de forma clara o objetivo do material educativo. ${ }^{26}$ Tais aspectos foram identificados pelos idosos e especialistas durante avaliação dos folhetos.

Além disso, o fornecimento de materiais educativos permite que eles sejam utilizados como ferramenta de auxílio didático, pois funcionam como um meio prático de acesso à informação. ${ }^{35}$ No presente estudo, os materiais fornecidos possibilitaram que os pacientes compreendessem as limitações da retirada gradual do medicamento e os benefícios da realização de mudanças nos hábitos de sono para melhor adaptação ao processo de desprescrição.

Como limitação deste estudo, destaca-se a restrição do protocolo ao clonazepam, em detrimento da inclusão de todos os medicamentos da classe dos benzodiazepínicos, uma vez que este grupo, como um todo, é amplamente prescrito e definido como potencialmente inapropriado para o uso em idosos. No entanto, este protocolo pode ser utilizado de forma adaptada para outros medicamentos da classe dos benzodiazepínicos por meio da conversão da dose, conforme proposto por Ashton. ${ }^{36}$ Além disso, já estão disponíveis na Internet algumas calculadoras de conversão de dose de um benzodiazepínico para qualquer outro. ${ }^{37}$

Assim, se um paciente se adequa aos critérios de inclusão e utiliza outro benzodiazepínico, que não o clonazepam, como medicação de uso contínuo, é possível realizar a desprescrição com o auxílio desses métodos de conversão de dose, através de duas formas: ou muda-se o benzodiazepínico utilizado para o clonazepam e segue-se as orientações do protocolo; ou encontra-se o valor equivalente a 0,5 mg do clonazepam em relação ao outro benzodiazepínico e segue-se a redução com este valor encontrado. Destacase que a decisão quanto a qual dos métodos escolher deve ser tomada de acordo com o julgamento clínico do médico assistente, o diálogo com o paciente e a disponibilidade dos medicamentos no serviço público.

Outra limitação do estudo diz respeito ao reduzido número de respostas na segunda etapa de avaliação. Considerando os valores absolutos, o número de respostas foi inferior, se comparado à primeira etapa do processo. Contudo, a proporção manteve-se em torno dos $25 \%$ relatados na literatura. ${ }^{20}$ Além disso, em ambas as etapas, o perfil predominante dos avaliadores foi de especialistas em Medicina de Família e Comunidade, o que pode ter influenciado no conteúdo das sugestões acerca do processo de validação, visto que as demais especialidades apresentam pontos de vista distintos em relação ao cuidado com o paciente e também são aptas a utilizar o protocolo na prática clínica.

Em adição, este protocolo possui um grande potencial para ser utilizado no sistema de saúde em todo território brasileiro, tanto no setor público como privado, uma vez que sua elaboração e validação foi fundamentada em evidências científicas atuais, experiências clínicas e características de cada setor. Ademais, o protocolo contempla a possibilidade de desprescrição nas duas formas de apresentação do medicamento e inclui medicações adjuvantes que estão disponíveis pelo SUS. 


\section{Conclusão}

A elaboração do protocolo mostrou-se relevante, uma vez que considerou as particularidades da população idosa, fundamentou-se em trabalhos da literatura em que o processo da desprescrição foi bem sucedido e contemplou todas as etapas necessárias para a retirada gradual do clonazepam. Os folhetos, por sua vez, ampliam a possibilidade de promoção do autocuidado e complementam o processo de desprescrição ao fornecer informações importantes de forma simples e objetiva ao paciente.

A validação do protocolo por meio da Técnica de Delphi foi pertinente, uma vez que, ao término da segunda etapa, todos os itens obtiveram o CVCc superiores a 0,8 , ponto de corte preconizado para que cada tópico fosse considerado válido.

Considerando os resultados obtidos, o protocolo se apresenta como uma ferramenta importante ao guiar a conduta médica no processo de desprescrição. Novos estudos envolvendo a execução do protocolo devem ser realizados com o intuito de avaliar sua viabilidade, aplicabilidade, reprodutibilidade e efetividade.

\section{Contribuição dos autores}

AOB, PFZ, LGV, MAAJ, ACLP, JVLN, IMB e TMR. Concepção e delineamento do trabalho: AOB, PFZ, LGV, MAAJ, ACLP, JVLN, IMB e TMR. Aquisição, análise ou interpretação dos dados: AOB, PFZ, LGV, MAAJ, ACLP, JVLN, IMB e TMR.

Revisão de versão preliminar: AOB, PFZ, LGV, MAAJ, ACLP, JVLN, IMB e TMR.

Aprovação final da versão a ser publicada: AOB, PFZ, LGV, MAAJ, ACLP, JVLN, IMB e TMR.

Todos os autores concordam em prestar contas de todos os aspectos do trabalho, assegurando que as questões relacionadas à acurácia ou integridade de qualquer parte do trabalho sejam devidamente investigadas e resolvidas.

\section{Referências}

1. Miranda GMD, Mendes ACG, Silva ALA. Population aging in Brazil: current and future social challenges and consequences. Rev Bras Geriatr Gerontol. 2016;19(3):507-19. DOI: http://dx.doi.org/10.1590/1809-98232016019.150140

2. Duarte ABS, de Macêdo ISV, de Assis KMA, Barros AES. Medicamentos inapropriados para idosos: uma revisão. In: 4 Congresso Internacional de Envelhecimento Humano; 2015 Set 21-26; Campina Grande, PB, Brasil. Campina Grande: Editora Realize; 2015.

3. Goldman L, Schafer AI. Goldman-Cecil: Medicina. 25aㅡ ed. Rio de Janeiro: Elsevier; 2018.

4. Manso MEG, Biffi ECA, Gerardi TJ. Inappropriate medication use in older adults with chronic diseases in a health plan in São Paulo, Brazil. Rev Bras Geriatr Gerontol. 2015;18(1):151-64. DOI: http://dx.doi.org/10.1590/1981-22562017020.160111

5. Oliveira MG, Amorim WW, Oliveira CRB, Coqueiro HL, Gusmão LC, Passos LC. Consenso brasileiro de medicamentos potencialmente inapropriados para idosos. Geriatr Gerontol Aging. 2016;10(4):168-81. DOI: 10.5327/Z2447-211520161600054

6. American Geriatrics Society 2015 Beers Criteria Update Expert Panel. American Geriatrics Society 2015 Updated Beers Criteria for Potentially Inappropriate Medication Use in Older Adults. J Am Geriatr Soc. 2015;63(11):2227-46.

7. Alvarenga JM, Loyola Filho Al, Giacomin KL, Uchoa E, Firmo JOA. Uso de benzodiazepínicos entre idosos: o alívio de "jogar água no fogo", não pensar e dormir. Rev Bras Geriatr Gerontol. 2015;18(2):249-58. DOI: http://dx.doi.org/10.1590/1809-9823.2015.14045

8. Pottie K, Thompson W, Davies S, Grenier J, Sadowski CA, Welch V, et al. Deprescribing benzodiazepine receptor agonists: Evidencebased clinical practice guideline. Can Fam Physician. 2018;64(5):339-51.

9. Reeve E, Gnjidic D, Long J, Hilmer S. A systematic review of the emerging definition of 'deprescribing' with network analysis: implications for future research and clinical practice. Br J Clin Pharmacol. 2015;80(6):1254-68. DOI: 10.1111/bcp.12732 
10. Griffin CE 3rd, Kaye AM, Bueno FR, Kaye AD. Benzodiazepine pharmacology and central nervous system-mediated effects. Ochsner J. 2013;13(2):214-23.

11. Ministério da Saúde (BR), Agência Nacional de Vigilância Sanitária (ANVISA). Panorama dos dados do sistema nacional de gerenciamento de produtos controlados: um sistema para o monitoramento de medicamentos no Brasil. Boletim de Farmacoepidemiologia. Brasília (DF): Anvisa; 2011. Disponível em: http://www.anvisa.gov.br/sngpc/boletins/2011/boletim_sngpc_2edatualizada.pdf

12. Ministério da Saúde (BR), Grupo Hospitalar Conceição. Gerência de Ensino e Pesquisa. Diretrizes Clínicas/Protocolos Assistenciais. Porto Alegre: Manual Operacional; 2008.

13. Parr JM, Kavanagh DJ, Cahill L, Mitchell G, Young R. Effectiveness of current treatment approaches for benzodiazepine discontinuation: a meta-analysis. Addiction. 2009;104(1):13-24. DOI:10.1111/j.1360-0443.2008.02364.x

14. Alvarenga JM, Giacomin KC, Loyola Filho Al, Uchoa E, Firmo JOA. Uso crônico de benzodiazepínicos entre idosos. Rev Saúde Pública. 2014;48(6):866-72. DOI: 10.1590/S0034-8910.2014048004986

15. Reeve E, Shakib S, Hendrix I, Roberts MS, Wiese MD. Review of deprescribing processes and development of an evidence-based, patient-centred deprescribing process. Br J Clin Pharmacol. 2014;78(4):738-47. DOI: 10.1111/bcp.12386

16. Baillargeon L, Landreville P, Verreault R, Beauchemin JP, Grégoire JP, Morin CM. Discontinuation of benzodiazepines among older insomniac adults treated with cognitive-behavioural therapy combined with gradual tapering: a randomized trial. CMAJ.2003;169(10):1015-20.

17. Voshaar RC, Couvée JE, van Balkom AJ, Mulder PG, Zitman FG. Strategies for discontinuing long-term benzodiazepine use: metaanalysis. Br J Psychiatry. 2006;189(3):213-20. DOI: 10.1192/bjp.189.3.213

18. Lader M, Tylee A, Donoghue J. Withdrawing benzodiazepines in primary care. CNS Drugs. 2009;23(1):19-34. DOI: 10.2165/0023210200923010-00002

19. Coluci MZO, Alexandre NMC, Milani D. Construção de instrumentos de medida na área da saúde. Ciênc Saúde Coletiva.2015;20(3):92536. DOI: $10.1590 / 1413-81232015203.04332013$

20. Marconi MA, Lakatos EM. Técnicas de pesquisa. $7^{\text {ạ }}$ ed. São Paulo: Editora Atlas; 2013.

21. Hernández-Nieto R. Contributions to statistical analysis. Mérida: Universidad de Los Andes; 2002.

22. Dias RCB. Método Delphi: Uma descrição de seus principais conceitos e características [Monografia]. São Paulo: Universidade de São Paulo; 2007.80 p.

23. Anacleto Junior MA. Protocolo de Desprescrição do Clonazepam em Idosos [Internet]. OSF; 2019. Disponível em: osf.io/drj52

24. Naloto DCC, Lopes FC, Barberato-Filho S, Lopes LC, Del Fiol FS, Bergamaschi CC. Prescrição de benzodiazepínicos para adultos e idosos de um ambulatório de saúde mental. Ciênc Saúde Coletiva. 2016;21(4):1267-76. DOI: http://dx.doi.org/10.1590/141381232015214.10292015.

25. Ministério da Saúde (BR). Portaria №2.436, de 21 de setembro de 2017. Aprova a Política Nacional de Atenção Básica, estabelecendo a revisão de diretrizes para a organização da Atenção Básica, no âmbito do Sistema Único de Saúde (SUS). Brasília (DF): Ministério da Saúde; 2017.

26. Hoffmann T, Warrall L. Designing effective written health education materials: considerations for health professionals. Disabil Rehabil. 2004;26(9):1166-73. DOI: 10.1080/09638280410001724816

27. Harrington HJ. Aperfeiçoando processos empresariais: estratégia revolucionária para o aperfeiçoamento da qualidade, da produtividade e da competitividade. São Paulo: Makron Books; 1993.

28. Farrell B, Tsang C, Raman-Wilms L, Irving H, Conklin J, Pottie K. What are priorities for deprescribing for elderly patients? Capturing the voice of practitioners: a modified Delphi process. PLoS One. 2015;10(4):e0122246. DOI: 10.1371/journal.pone.0122246

29. Nakao M, Takeuchi T, Nomura K, Teramoto T, Yano E. Clinical application of paroxetine for tapering benzodiazepine use in non-major-depressive outpatients visiting an internal medicine clinic. Psychiatry Clin Neurosci.2006;60(5):605-10. DOI: 10.1111/j.1440-1819.2006.01565.x

30. Brett J, Murnion B. Management of benzodiazepine misuse and dependence. Aust Prescr. 2015;38(5):152-5. DOI: 10.18773/austprescr.2015.055

31. Pasquali L. Psicometria: teoria dos testes na psicologia e na educação. 1ํㅡㄹ ed. Petrópolis: Vozes; 2017.

32. Pedreira RBS, Rocha SV, Santos CA, Vasconcelos LRC, Reis MC. Validade de conteúdo do instrumento de avaliação da saúde do idoso. Einstein (São Paulo). 2016;14(2):158-77. DOI: 10.1590/S1679-45082016AO3455 
33. Marques JBV, Freitas D. Método DELPHI: caracterização e potencialidades na pesquisa em Educação. Pro-Posições. 2018;29(2):389415. DOI: http://dx.doi.org/10.1590/1980-6248-2015-0140

34. Lima ACMACC, Bezerra KC, Sousa DMN, Rocha JF, Oriá MOB. Construção e validação de cartilha para prevenção da transmissão vertical do HIV. Acta Paul Enferm. 2017;30(2):181-9. DOI: http://dx.doi.org/10.1590/1982-0194201700028

35. Oliveira SC, Lopes MVO, Fernandes AFC. Construção e validação de cartilha educativa para alimentação saudável durante a gravidez. Rev Lat Am Enferm. 2014;22(4):611-20. DOI: 10.1590/0104-1169.3313.2459

36. Ashton $\mathrm{H}$. The treatment of benzodiazepine dependence. Addiction. 1994;89(11):1535-41.

37. Benzodiazepine Equivalents Conversion Calculator [Internet]. ClinCalc.com; 2017 [acesso 2019 Set 25]. Disponível em: https://clincalc. com/Benzodiazepine 